\title{
Stable quark stars beyond neutron stars: can they account for the missing matter?
}

\author{
Sonja Kabana* ${ }^{*}$ \\ Laboratory for High Energy Physics, University of Bern, Sidlerstrasse 5, 3012 Bern, \\ Switzerland \\ E-mail: isonja.kabana@cern.ch

\section{Peter Minkowski} \\ Institute for Theoretical Physics, University of Bern, Sidlerstrasse 5, 3012 Bern, \\ Switzerland \\ E-mail: 'mink@itp.unibe.ch'
}

\begin{abstract}
The structure of a spherically symmetric stable dark 'star' is discussed, at zero temperature, containing 1) a core of quarks in the deconfined phase and antileptons 2) a shell of hadrons in particular $n, p, \Lambda$ and $\Sigma^{-}$and leptons or antileptons and 3) a shell of hydrogen in the superfluid phase. If the superfluid hydrogen phase goes over into the electromagnetic plasma phase at densities well below one atom / $(10 \mathrm{fm})^{3}$, as is usually assumed, the hydrogen shell is insignificant for the mass and the radius of the 'star'. These quantities are then determined approximatively : mass $=1.8$ solar masses and radius $=9.2 \mathrm{~km}$. On the contrary if densities of the order of one atom $/(10 \mathrm{fm})^{3}$ do form a stable hydrogen superfluid phase, we find a large range of possible masses from 1.8 to 375 solar masses. The radii vary accordingly from 9 to $1200 \mathrm{~km}$.
\end{abstract}

KEywords: Dark Matter, Quark starsi.

\section{Introduction}

Many authors have found solutions for strange quark stars with shells of hadronic composition [i] outlined in the abstract. We use for the quark core the Tolman solution VI [2]2] with infinite central energy density and pressure. We also introduce a superfluid hydrogen shell which can be very large depending on its inner energy density. For normal hydrogen densities the hydrogen shell is very thin and negligible in mass. Objects with a mass of few solar masses can in principle be detected by gravitational lensing. However, in case of abnormally large

${ }^{*}$ Speaker.

${ }^{\dagger}$ We thank the Schweizerischer Nationalfonds for its support. 


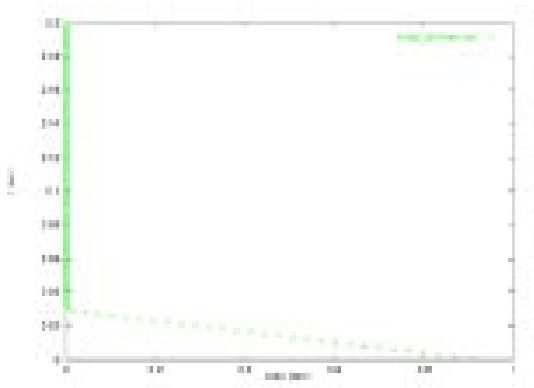

Figure 1: Temperature in $\mathrm{GeV}$ as a function of the baryochemical potential $\mu_{B}$ in $\mathrm{GeV}$, showing the path followed by the early universe after the QCD phase transition.

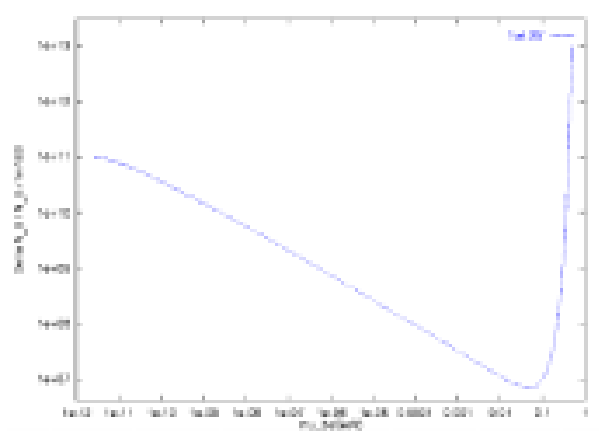

Figure 2: Relativ fluctuations of the net baryon number normalized to a volume of 1 $\mathrm{fm}^{3}$ as a function of the baryochemical potential, following the path of figure 1

initial energy density of the hydrogen shell, we arrive at very large masses of the star, which can not be easily detected by lensing. In this case, such stars are candidates for 'nonbaryonic' dark matter. The three shells are discussed in the following section. For details of this analysis see reference [i] $\left[\begin{array}{l}3 \\ ]\end{array}\right]$.

\section{The shells of the star}

We consider 3 shells of radii r0 (quark shell), r1 (hadron shell), and r2 (hydrogen shell). The quark core of the star is described according to Tolman's solution VI [2]2] and energy density and pressure are given by

$\rho_{e}=\frac{3}{56 \pi G_{N}} \frac{1}{r^{2}}$ and $p=\frac{1}{56 \pi G_{N}} \frac{1}{r^{2}} \frac{(1-9(B / A) r)}{(1-(B / A) r)}$.

Where $\mathrm{A}, \mathrm{B}$ are integration constants. We consider the core made up of $\mathrm{u}, \mathrm{d}, \mathrm{s}$ quarks and antileptons. allowing for the three chemical potentials associated with charge $\left(\mu_{Q}\right)$, baryon number $\left(\mu_{B}\right)$ and total lepton number $\left(\mu_{L}\right)$. The hadronic phase is composed of baryons, leptons and antileptons. We use in the hadronic phase the vacuum pressure from [i] The hydrodynamic and QCD equilibrium conditions between the two phases (hadronic and plasma) together with the condition of vanishing overall electric charge determine the core radius, mass and all chemical potentials for charge, baryon number and lepton number. We derive the mass $M$ and the radius $r 0$ of the core: $\mathrm{M}$ (quark core) $=0.457$ solar masses and $\mathrm{r} 0$ (quark core) $=3.149 \mathrm{~km}$. The baryon number of the core is

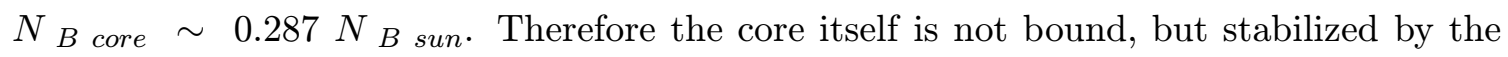
outer hadron shell. We simplify the hadron shell composition structure by considering only neutrons, protons and electrons, though near the QCD transition zone also $\Sigma^{-}, \Lambda, \mu^{+}, e^{+}$ and all antineutrinos intervene. We obtain : $M$ ( hadronic shell ) $=1.344$ solar masses and $r 1$ ( hadronic shell $)=9.047 \mathrm{~km}$. Therefore the total mass of both phases inside the radius $\mathrm{r} 1$ is: $M$ ( total inside $\mathrm{r} 1)=1.801$ solar masses and the baryon number inside $\mathrm{r}=\mathrm{r} 1$ is $1.853 N_{B}($ sun $)$. The nonrelativistic approximation of the TOV equation used here overestimates somewhat both mass and radius. We proceed to calculate the mass and radius of the hydrogen shell which starts at radius $\mathrm{r} 1$ and ends at radius $\mathrm{r} 2$. We use here the original approximate relations for superfluid hydrogen ${ }^{1}$, or alike boson-condensed

\footnotetext{
${ }^{1}$ We do not include any further light elements, fermions or bosons, in the hydrogen shell.
} 
non relativistic system, first described by N. N. Bogolubov [ip]. The results dependent on the parameter $\mathrm{H}$, which is the initial energy density of hydrogen at $\mathrm{r}=\mathrm{r} 1$, normalized to $\alpha m_{e} m_{p} m_{H}^{2}$. The total radii, masses and baryon numbers are shown in table 1 together with $\mathrm{H}$. The normal hydrogen shell corresponds to the first line.

\begin{tabular}{|c|c|c|c|}
\hline$H$ & $\begin{array}{c}\text { Radius of dark 'star' } \\
(\mathrm{km})\end{array}$ & $\begin{array}{c}\text { Mass of dark 'star' } \\
\text { (solar masses) }\end{array}$ & $\begin{array}{c}N_{B} \\
\left(N_{B}(\text { sun })\right)\end{array}$ \\
\hline $510^{-3}$ & 9.204 & 1.801 & 1.853 \\
\hline 0.290 & 411 & 2.191 & 2.243 \\
\hline 0.295 & 674 & 3.179 & 3.232 \\
\hline 0.310 & 1061 & 12.63 & 12.68 \\
\hline 0.50 & 1203 & 168.13 & 168.18 \\
\hline 0.625 & 1208 & 271.06 & 271.11 \\
\hline 0.75 & 1210 & 374.02 & 374.07 \\
\hline
\end{tabular}

Table 1: Mass and radius for the complete 'star' with a quark (anti)lepton core, a hadron shell and a hydrogen shell. $\mathrm{H}$ is the energy density of the hydrogen shell at $\mathrm{r}=\mathrm{r} 1$ normalised to $\rho_{e 1}=$ $\alpha m_{e} m_{p} m_{H}^{2}$.

\section{The big annihilation}

We follow the path of the early universe right after the QCD phase transition which is important for the genesis of quark stars. In particular fig. 11' shows the temperature as a function of the baryochemical potential. At about $\mathrm{T} \sim 30 \mathrm{MeV}$ the annihilation of matter and antimatter begins. We denote this 'the big annihilation'. Note that laboratory experiments measured a copious production of antibaryons at a temperature of $\sim 170 \mathrm{MeV}$ see e.g. $[\bar{i}]$. The statistical fluctuations of net baryon number normalized to a volume of $1 \mathrm{fm}^{3}$ following this path, are shown in fig. ${ }_{2 i}^{-i}$. They show maxima at the phase boundary and at temperatures below $10 \mathrm{MeV}$. The rest of the condensation of such a star needs large gravitational fluctuations too.

\section{Conclusions}

We investigated the structure of a spherically symmetric stable dark 'star'. For a low density hydrogen shell we find for the mass and the radius of the 'star' mass $=1.8$ solar masses and radius $=9.2 \mathrm{~km}$. For a high density hydrogen shell supporting densities of up to one atom / $(10 \mathrm{fm})^{3}$ we find a large range of possible masses from 1.8 to 375 solar masses. The radii vary accordingly from 9 to $1200 \mathrm{~km}$. Such objects are candidates for dark matter of any kind, despite their clearly baryonic nature, if they have been formed in such a way that they do not affect nucleosynthesis and the cosmic microwave background fluctuations at decoupling. We have calculated the path of the early universe after the QCD phase transition in the $\left(\mathrm{T}, \mu_{B}\right)$ plane, and the net baryon number fluctuations in the absence of gravitational fluctuations. We found that the statistical net baryon number fluctuations are large at the QCD phase transition at $\mu_{B} \sim 0$ and at $\mathrm{T} \sim 10 \mathrm{MeV}$ and less and at high $\mu_{B}$. These are regions where such stars could be formed. The mass dominating part of the hadron shell is very similar to a neutron star. This can be seen in our estimate of the mass : 
$\sim 1.34$ solar masses, slightly below the Chandrasekhar bound. The distinctive feature to a neutron star is that the hadron shell does not extend to the center of the 'star'. The quark core taken for itself and according to Tolmans universal solution, with $\rho_{e} \propto r^{-2}$ towards the center is not stable. This means that it contains less nucleons counted by number than counted by mass. Yet this does not imply any instability of the 'star' as a whole, as it would be the case for a one-phase neutron star. The phase boundary between quark core and hadron shell with its surface pressure stabilizes the 'star'. Despite genuine baryonic origin, besides the (anti)leptonic parts, such 'stars' are indeed candidates for any kind of dark matter. If 1.8 solar masses indeed characterize the mass of these 'stars' then they could be observed through gravitational microlensing of the galactic halo towards the Mag-

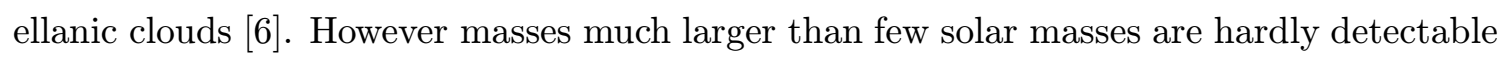
by lensing. We also note, that the composition of the 'star' considered here lacks heavy elements and hence the metallicity nearby is unusually low compared to burning stars of similar mass. They could possibly emit and absorb $\mathrm{H}$ lines, e.g. Lyman $-\alpha$ lines taking into account gravitational redshift (which is $\mathrm{z}=0.56$ for a mass of 1.8 solar masses) and high pressure compared to normal conditions. Another possibility is that they are part of a gravitating system including one or more shining stars.

\section{Acknowledgments}

We thank Dr. J. Schaffner-Bielich, Dr. R. von Steiger and Prof. K. Pretzl for discussions.

\section{References}

[1] F. Weber, J. Phys. G27 (2001) 465, astro-ph/0008376, H. Huber et al., Phys. Rev. C57 (1998) 3484, nucl-th/9803026, J. Madsen, Phys. Rev. Lett. 85 (2000) 10, astro-ph/9912418, astro-ph/9809032, J. A. Pons et al., astro-ph/0107404, M. Prakash et al., astro-ph/0012136, J.M. Lattimer and M. Prakash, Astrophys. J. 550 (2001) 426, astro-ph/0002232.

[2] R.C. Tolman, Phys. Rev. 55 (1939) 364.

[3] P. Minkowski, S. Kabana, proceedings of the workshop on 'Matter in the universe', ISSI, Bern, March 2001, to appear in Space Science Reviews, Kluwer Acedemic Publishers, the Netherlands.

[4] S. Kabana and P. Minkowski, New. J. Phys. 3 (2001) 4.

[5] N. N. Bogolubov, Journal of Physics USSR, XI (1947) 23.

[6] Ph. Jetzer, astro-ph/9901058, proceedings of EC-TMR Euroconference on 3K Cosmology, Rome, Italy, 5-10 Oct 1998.

[7] G. Ambrosini et al., (NA52 coll.): nucl-ex/0011016, Phys. Lett. B 417 (1998) 202, New J. of Phys. 1 (1999) 22, T. Alber et al., Phys. Lett. B 366 (1996) 56, C. Adler et al., (STAR coll.), nucl-ex/0108022. 